Ann. Biol. anim. Bioch. Biophys., I962, 2 (2), I67-I74

\title{
ACTION DE L'ABLATION DES BULBES OLFACTIFS SUR LES MÉCANISMES DE LA REPRODUCTION CHEZ LA TRUIE
}

\author{
J. P. SIGNORLT et P. MAULEON \\ avec la collaboration technique de Colette LAvenet \\ Station de Recherches de Physiologie animale, \\ Centre national de Recherches zoolechniques, Jouy-en-Josas (Seine et Oise).
}

\section{SOMMAIRE} White.

L'ablation chirurgicale des bulbes olfactifs a été effectuée chez i 5 jeunes truies de type Large

5 d'entre elles ont des cycles réguliers et un tractus génital normal à la suite d'une ablation que la dissection a révélée n'être que partielle.

Les io autres ont présenté d'importantes perturbations :

Pour 6 d'entre elles il s'agissait d'un anœestrus permanent accompagné d'une forte involution du tractus génital et d'une mise au repos des ovaires. L'étude histologique de l'hypophyse a montré une accumulation de matériel sécrétoire (cellules $\beta$ et $\gamma$ ). L'ablation était totale.

Les 4 autres ont présenté des périodes anœstrus de durée variable sans involution utérine.

L'ablation totale des bulbes olfactifs semble donc perturber la décharge des hormones gonadotropes par l'hypophyse.

Des résultats récents ont mis en évidence une action nette de stimuli olfactifs sur les mécanismes de reproduction chez la femelle, une revue de cette question a été publiée par PARKES et BRUCE (I96r). Nous avons entrepris l'étude de ces mécanismes en agissant directement sur les structures nerveuses de l'olfaction.

Les résultats obtenus par divers auteurs à la suite de l'ablation des bulbes olfactifs sont assez discordants. Seul WHITTEN (I956) constate chez les souris, à la suite d'une ablation totale des bulbes une forte régression des ovaires et de 1'utérus malheureusement l'étude histologique des ovaires et de 1'hypophyse n'a pas été effectuée. Ceci est à rapprocher de l'analyse faite par de MoRSIER (I954) : passant en revue les 
cas d'absence congénitale des bulbes olfactifs chez l'homme, il relève une liaison très fréquente entre cette anomalie et un infantilisme génital.

Par contre plusieurs auteurs ont observé des ovulations ou des gestations normales à la suite de l'ablation totale des bulbes olfactifs chez la souris (LAMOND, I958) et la lapine (Stone, I925 ; Brooks, I937 ; KöHNE, I947 ; SAWyer, I956-I959). Cependant ce dernier en I955 a obtenu à la suite de l'ablation des bulbes un blocage de l'ovulation déclenchée par l'injection intraventriculaire d'histamine.

D'autre part LAMOND a obtenu un fort pourcentage d'infécondité et KöHNE comme SAWYER ont observé parmi leurs animaux opérés des cas d'involution utérine et ovarienne.

Les résultats obtenus à la suite de l'ablation des bulbes olfactifs sont rapportés ici.

\section{MATÉRIEL ET MÉTHODES}

\section{Animaux}

Nous avons utilisé I 5 jeunes truies pubères de type Large White n'ayant jamais porté. Le cycle sexuel est déterminé en présentant deux fois par jour toutes les femelles, par groupe de 5 ou 6 , ̀̀ un mâle.

\section{Technique chirurgicale}

Les aninnaux, opérés à un poids variant entre roo et $\mathrm{r} 20 \mathrm{~kg}$, sont anesthésiés par injection intraveineuse de chloral ; pendant l'opération l'anesthésie est entretenue par la respiration d'un mélange protoxyde-oxygène-trichloréthylène.

La voie d'accès des bulbes olfactifs est indiquée par la figure I :

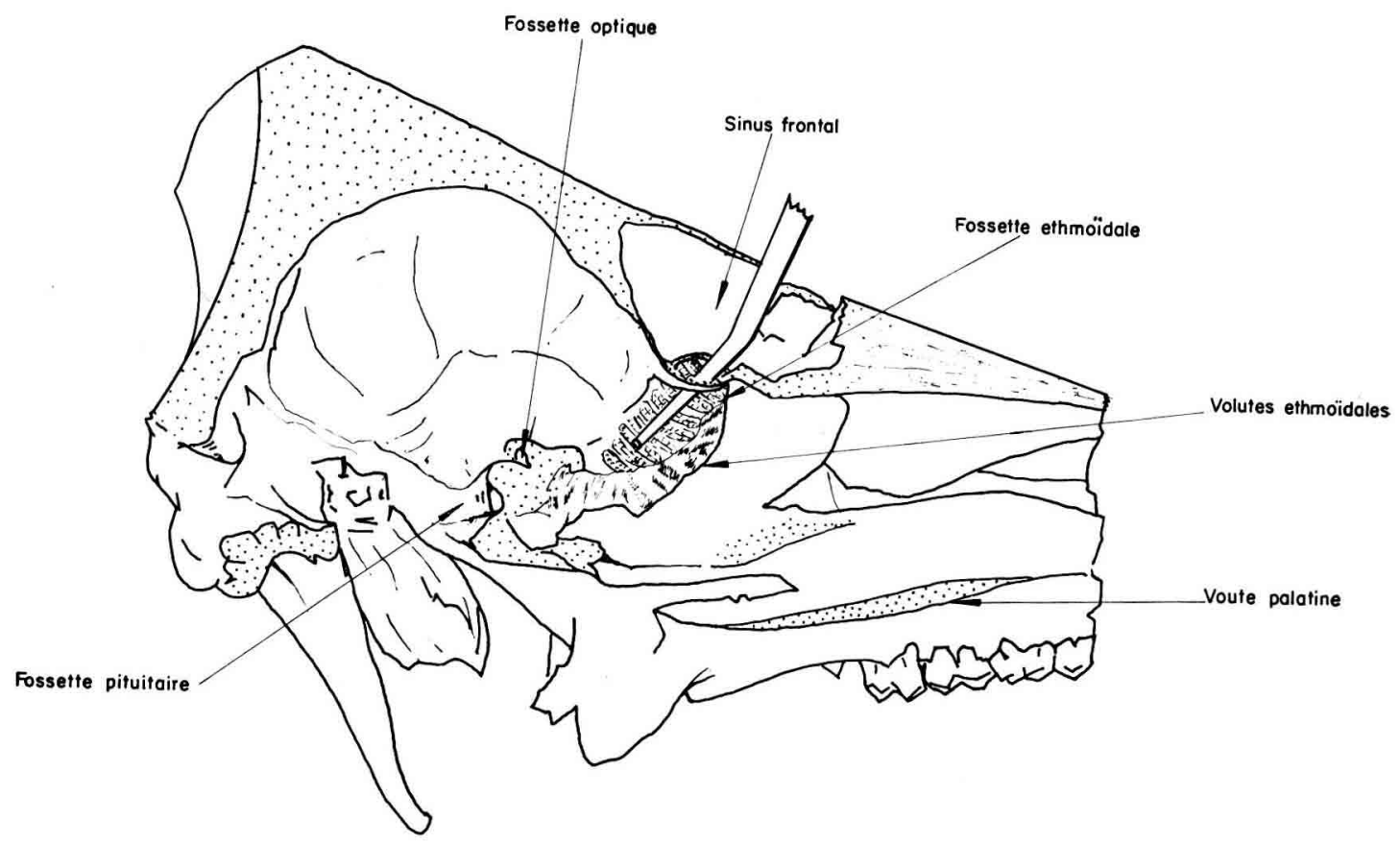

FIG. I - Voie d'accès chirurgicale des bulbes olfactifs 
La paroi osseuse recouvrant les bulbes olfactifs est abordée par les sinus frontaux et perforée au moyen d'une fraise. Le bulbe olfactif est alors enlevé par succion, l'hémorragie est arrêtée en introduisant à plusieurs reprises dans la cavité laissée libre à la suite de l'ablation, de l'éponge de gélatine imbibée de thrombase. La dernière application est laissée en place. Le périoste et la peau sont suturés séparément. La récupération postopératoire est très rapide. Nous n'avons pas préparé d'animaux témoins opérés sans ablation.

\section{Abattage et prélèvements}

Les truies sont assommées par un choc électrique puis saignées. La tête est immédiatement coupée puis perfusée au sérum physiologique d'abord, puis avec une solution de formol à ro p. Ioo pour la fixation des tissus.

Le résultat de l'ablation est contrôlé par une dissection soigneuse de la partie opérée.

\section{Histologie}

Les hypophyses fixées au formol-sublimé sont colorées selon la technique de IIERLANT (I958) (PAS-Bleu Alcian).

Les ovaires sont fixés au Bouin, leur structure est étudiée après coloration au trichrome de Masson.

\section{RÉSULTATS}

\section{L'ensemble des résultats est présenté dans le tableau $\mathrm{I}$.}

TABLFAU I

Influence de l'ablation des lobes clfactifs sur les cycles sexuels et le tractus génital

\begin{tabular}{|c|c|c|c|c|c|c|}
\hline $\begin{array}{l}\text { Résultat } \\
\text { de } \\
\text { l'ablation }\end{array}$ & $\begin{array}{l}\text { État } \\
\text { des } \\
\text { truies }\end{array}$ & $N^{\circ}$ truie & $\begin{array}{l}\text { Cycles postopératoires } \\
\text { jusqu'à l'abattage }\end{array}$ & Type d'ovaire & cort & $\begin{array}{l}\text { Poids des } 2 \\
\text { mes utérines en } \\
\text { grammes }\end{array}$ \\
\hline \multirow[b]{2}{*}{ partielle } & normales & $\begin{array}{l}1467 \\
1508 \\
1488 \\
1578 \\
1556\end{array}$ & $\begin{array}{l}3 \text { cycles normaux } \\
4 \text { cycles normaux } \\
4 \text { cycles normaux } \\
5 \text { cycles normaux } \\
4 \text { cycles normaux }\end{array}$ & $\begin{array}{c}\text { corps jaunes } \\
\text { follicules matures } \\
\text { corps jaunes }\end{array}$ & $\left|\begin{array}{l}309 \\
511 \\
496 \\
392 \\
423\end{array}\right|$ & Moy. $=426,2$ \\
\hline & perturbées & $\begin{array}{l}14+73 \\
1484\end{array}$ & $\begin{array}{l}1 \text { cycle normal, } 63 \mathrm{j} \\
\text { d'anosstrus et abattage } \\
\text { en début de chaleur } \\
3 \text { cycles normaux, puis pas } \\
\text { de chaleurs nettes pendant } \\
6: 3 \mathrm{j}\end{array}$ & $\begin{array}{l}\text { foll. matures, pas de } \\
\text { trace de c. j. en } \\
\text { régression } \\
\text { pas de c. j. regressés } \\
\text { mais dévelop. foll. } \\
\text { en cours }\end{array}$ & $\begin{array}{l}465 \\
300\end{array}$ & \\
\hline \multirow{2}{*}{$\begin{array}{l}\text { subtotale } \\
\text { ou totale }\end{array}$} & & $\begin{array}{l}1499 \\
1495\end{array}$ & $\begin{array}{l}2 \text { cycles normaux, puis } \\
\text { pas de chaleur nette } \\
\text { pendant } 102 \mathrm{j} \\
2 \text { cycles normaux, } 42 \mathrm{j} \\
\text { repos, } 1 \text { ostrus, puis } \\
25 \mathrm{j} \text { sans chaleur }\end{array}$ & $\begin{array}{l}\text { c. j. en activité } \\
\text { (j)seudo-gestation?) } \\
\text { c. j. regressés et } \\
\text { petits follicules }\end{array}$ & $\begin{array}{l}546 \\
365\end{array}$ & \\
\hline & ancestrus & $\begin{array}{l}1504 \\
1609 \\
1604\end{array}$ & $\begin{array}{l}\text { aucun cycle pdt } 103 \mathrm{j} \\
\text { aucun cycle pdt } 55 \mathrm{j} \\
2 \text { cycles normaux puis } 86 \mathrm{j} \\
\text { sans chaleur } \\
1 \text { cycle puis } 95 \mathrm{j} \text { sans } \\
\text { chaleur } \\
1 \text { cycle puis } 65 \mathrm{j} \text { sans } \\
\text { chaleur } \\
2 \text { cycles normaux puis } 90 \mathrm{j} \\
\text { saus chaleur }\end{array}$ & $\begin{array}{l}\text { petits follicules } \\
\text { petits follicules } \\
\text { petits follicules } \\
\text { petits follicules } \\
\text { petits follicules } \\
\text { petits follicules }\end{array}$ & $\left|\begin{array}{r}163 \\
107 \\
133 \\
67 \\
72 \\
131\end{array}\right|$ & Moy. $=112,0$ \\
\hline
\end{tabular}




\section{Action sur les cycles sexuels}

Après l'opération la majorité des animaux, ro sur $\Upsilon_{5}$, a présenté des modifications très importantes dans le déroulement des cycles sexuels.

Chez 2 animaux aucun ostrus n'est réapparu après 1'opération.

Chez 6 autres truies, après un à trois cycles normaux on a observé un arrêt complet des manifestations sexuelles jusqu'à l'abattage après une période de 2 à 3 mois. 2 truies ont présenté à côté de cycles normaux des périodes d'anœstrus.

Enfin les 5 autres animaux ont été abattus après 3 à 5 cycles normaux.

\section{Action sur le tractus génital et les ovaires}

A l'abattage nous avons pu constater que ces perturbations des manifestations sexuelles cycliques n'étaient pas dues uniquement à des modifications du comportement mais se répercutaient également au niveau du tractus génital et des ovaires.

On peut de ce point de vue répartir nos animaux en deux groupes :

- utérus involué (poids inférieur à $200 \mathrm{~g}$ ) ;

- utérus normal (poids égal ou supérieur à $300 \mathrm{~g}$ ).

Dans le premier cas les ovaires ne présentaient aucun corps jaune récent ou regressé visible ; ils étaient caractérisés par la présence exclusive de follicules de petite dimension (diamètre $2 \mathrm{~mm}$ ), comparables aux ovaires des animaux impubères (voir figure 2 ).

Dans le deuxième cas chaque animal sera examiné individuellement.

- La truies $n^{\circ}$ I 484 après 3 cycles normaux a présenté un anœstrus de plus de 60 jours jusqu'à l'abattage, l'ovaire ne présentait aucune trace de corps jaunes récents ou anciens mais il semble que le fonctionnement ovarien ait été à la veille de reprendre puisque l'on pouvait constater une maturation folliculaire en cours.

- La truie no $n^{\circ} 473$ est un cas identique, mais elle était venue en ostrus le jour même de l'abattage.

- La truie $n^{\circ}$ I495 après un anœstrus de 42 jours interrompu par un œstrus avec ovulation semblait au moment de l'abattage retomber en repos ovarien comme le montre l'absence de stimulation folliculaire.

- La truie $n^{\circ}$ I499 est un cas particulier puisqu'après Io2 jours sans manifestation sexuelle, ses ovaires ont été trouvés porteurs de corps jaunes en activité suggérant l'idée d'une pseudo-gestation.

\section{Action au niveau hypophysaive}

Pour aborder l'étude du mécanisme des perturbations constatées, nous avons analysé par les méthodes histochimiques l'état de 1'hypophyse des animaux expérimentaux lors de 1'abattage. La technique de HERLANT (I958) (PAS-BLEU Alcian) permet de différencier trois catégories cellulaires :

- les cellules $\beta$ dont la teneur en FSH est mise en évidence par une coloration bleue violacé.

- les cellules $\gamma$ dont la teneur en $\mathrm{LH}$ est mise en évidence par une coloration rose vif ;

- les cellules $\delta$ dont la teneur en TSH est mise en évidence par une coloration bleu franc. 

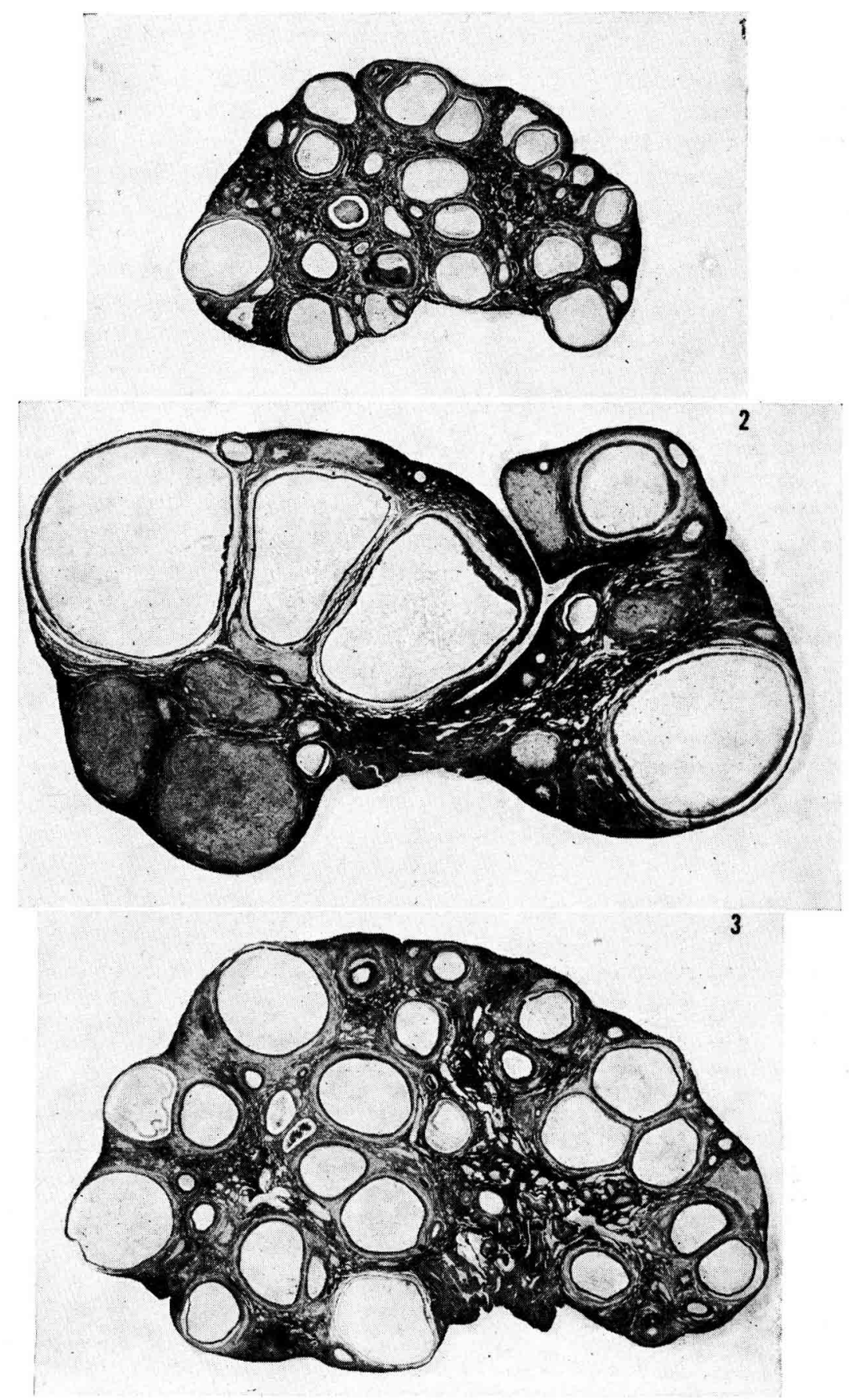

Fig. 2. - Coupes d'ovaires de truies

I : truie impubère.

2 : truie $\mathrm{n}^{0}{ }_{1578}$ cycles normaux - ovaires prélevés en phase folliculaire.

3 : truie $n^{0}$ r 47 I cycles bloqués - ovaires prélevés 2 mois après l'ablation des bulbes olfactifs.

Remarquer dans l'ovaire de la truie 147 I (3) l'accumulation de follicules qui ne dépassent pas un diamètre de $2 \mathrm{~mm}$ donnant ainsi une population comparable à celle caractéristique de la truie impubère (I). 
Dans les hypophyses des truies no $n_{504}$ et I609 dont le tractus génital est fortement involué, il y a une accumulation considérable dans tout le tissu de cellules $\beta$ et $\gamma$ riches en produits colorables.

Dans les hypophyses des truies no I488 et I 467 ayant eu des cycles normaux les cellules $\beta$ sont beaucoup plus rares et les cellules $\gamma$ restent localisées à certaines zones (voir figure 3 ).

Ces images hypophysaires suggèrent un blocage de la décharge de gonadotrophines chez les truies sans cycle puisqu'il y a accumulation de matériel sécrétoire dans les cellules.

\section{Contrôle de l'ablation}

Nous avons donc obtenu chez 6 truies une involution complète du tractus ainsi qu'une mise au repos des ovaires. Le contrôle effectué après 1'abattage a montré que chez tous ces animạx l'ablation des bulbes olfactifs était totale. Par contre chez les 5 truies ayant eu une succession de cycles normaux l'ablation n'était que partielle.

L es animaux dont 1'ensemble des manifestations cycliques a été perturbé se partageaient entre ces deux résultats opératoires (voir tableau I).

En conclusion, il semble donc que l'ablation totale des bulbes olfactifs provoque chez la truie un arrêt de la décharge des hormones gonadotropes entraînant une absence de maturation folliculaire et d'ovulation, une involution de l'utérus et un arrêt des manifestations sexuelles.

A la suite d'ablations partielles, on peut voir soit un fonctionnement normal soit des perturbations de moindre importance.

\section{Reģu en åril 1962.}

\section{SUMMARY}

THE EFFECT OF SURGICAL REMOVAL OF THE OLFACTORY BULBS ON THE SEXUAL CYCLE AND TIFE GENITAL TRACT OF SOWS

Surgical removal of the olfactory bulbs has been performed in ${ }_{5}$ Large White gilts.

-5 gilts exhibited a number of normal cycles, the uteri and ovaries were normal; autopsy revealed residual portions of the bulbs in all cases.

-6 gilts were in permanent anœstrus. Uterine weights were significantly reduced $(\mathrm{P}<0.0 \mathrm{I})$ and the ovaries were similar to these of prepubertal animals. Histochemical study of the hypophysis showed an accumulation of secretory material in the $\beta$ and $\gamma$ cells. Removal of the bulbs was complete.

4 gilts showed periods of anœstrus without uterine involution.

Complete removal of the olfactory bulbs seems to interfere with the discharge of pituitary gonadotrophins.

\section{RÉFÉRENCES BIBLIOGRAPHIQUES}

Brooks C. Mc. MC., I937. The role of the cerebral cortex and of various sense organs in the excitation and execution of mating activity in the rabbit. Amer. J. Physiol., 120, 544-553.

Herlant M., 1958. Les glycoprotéines du lobe antérieur de l'hypophyse. Ann. Histochim., 3, 67-83.

KöHNE G., 1947. Die Beziehungen der angeborenen Olfaktoriusdefekts zum primären Eunuchoidismus des Mannes. Virchow's Arch., 314, 345.

LAMOND D. R., I958. Infertility associated with extirpation of the olfactory bulbs in female albino mice. Aust. J. exp. biol. med. sci., 36, 103-109.

Morsier G. de, I954. Etudes sur les dysraphies cranio-encéphaliques. I - Agenesie des bulbes olfactifs et des commissures calleuse et antérieure. Schweiz. Arch. neurol. Psychiat., 74, 309-36r. 

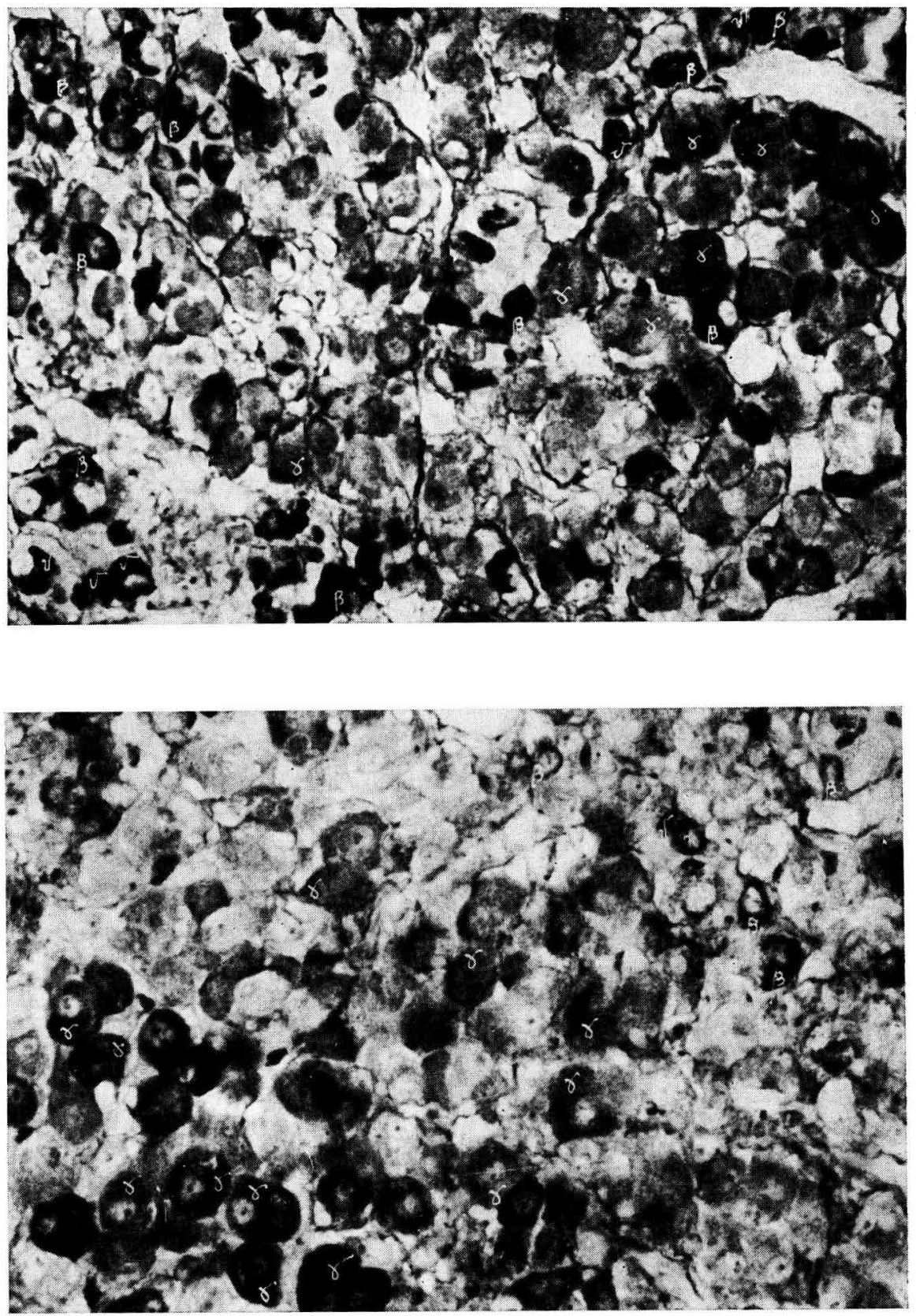

FIG. 3. - Coupes d'hypophyse coloration (PAS-Bleu Alcian) I : truie $n^{\circ} 1504$ cycles bloqués.

II : truie $n^{0}$ I 488 cycles normaux.

Remarquer l'abondance des cellules $\beta$ de petite tai le ainsi que des cellules $\gamma$ à la suite da l'ablation totale des bulbos olfactifs, donnant une accumulation considérable də matériel sécrétoire colorable. 
PARkes A. S. Bruce H. M., 196r. Olfactory stimuli in mammalian reproduction. Odor excites neurohumoral responses affecting cstrus, pseudopregnancy and pregnancy on the mouse. Science, 134, 1049-1054. SAWYER C. H., I955. Rhinencephalic involvement in pituitary activation by intraventricular histamine in the rabbit under nembutal anesthesia. Amer. J. Physiol., 180, 37-46.

SaWyer C. H., I956. Effect of central nervous system lesions on ovulation in the rabbit. Anat. Rec., 124, 358 .

SAWYER C. H., I959. Effects of brain lesions on estrous behavior and reflexogenous ovulation in the rabbit. J. Exp. Zool., 142, 227-246.

STONE C. P., 1925. The effect of cerebral destruction on the sexual behavior of rabbits. I - The olfactory bulbs. Amer. J. Physiol., 71, 430-435.

Whitren W. K., I956. The effect of removal of the olfactory bulbs on the gonads of mice. J. Endocrinol. 14, $160-163$. 